BASHH

\section{BASHH guideline for gonorrhoea}

\section{J Bignell}

\section{Time for change}

S tandards for the management of gonorrhoea need to encompass relief of symptoms, rapid eradication of Neisseria gonorrhoeae from all sites, action to ensure that sexual partners are treated, and sexual health education to promote risk reduction in future sexual interaction. Many antimicrobial regimens have in the past demonstrated the capacity to eradicate $N$ gonorrhoeae from genital sites. ${ }^{1}$ Efficacy in clinical trials has been shown to correlate with the length of time a particular antimicrobial regimen provides a blood level of four times the $\mathrm{MIC}_{90} \cdot{ }^{12}$ Sensitivity and pharmacokinetics are therefore key factors, together with data from clinical trials and toxicity, in determining treatment recommendations. Weighing these factors is particularly relevant to the choice of recommended cephalosporins. ${ }^{3}$ The prevalence of gonococcal resistance to antimicrobials is variable and can change dramatically. Pharmacokinetics can differ significantly within and between classes of antimicrobials. It is therefore not surprising that antimicrobial regimens differ in clinical efficacy, particularly when eradication from nongenital sites is considered. What worked in treating gonorrhoea yesterday or even today will not necessarily continue to be an effective treatment.

While all guidelines need regular review, the British Association of Sexual Health and HIV (BASHH)/ National guideline on gonorrhoea ${ }^{4}$ has needed a significant change in recommended therapy in response to data from the Gonococcal Resistance to Antimicrobial Programme (GRASP), which has shown a dramatic rise in high level quinolone resistance in England and Wales during the past 2 years. ${ }^{56}$ In 2001, the majority of people with gonorrhoea attending genitourinary medicine clinics in the United Kingdom received a quinolone antibiotic, consistent with the former guideline. ${ }^{7}$ Quinolone therapy alone would now result in failure to resolve symptoms or eradicate $N$ gonorrhoeae in more than $10 \%$ of unselected cases in some regions, although clinical failures have been uncommon because treatment for gonorrhoea is frequently combined with a tetracycline or macrolide to manage possible co-incidental chlamydial infection. When a patient is identified to have gonorrhoea in the United Kingdom, it can now be anticipated that resistance to penicillins, tetracyclines, and quinolones will all exceed $5 \%{ }^{5}$

Options are now limited to achieve a minimum of $95 \%$ cure of unselected cases of anogenital gonorrhoea with single dose antimicrobial therapy at initial presentation. Treatment trials for gonorrhoea have been few in recent years and the revised 2004 recommendation to use a long acting third generation cephalosporin, notably ceftriaxone or cefixime, is based on historical efficacy from clinical trials, the known pharmacokinetics of these antimicrobials, in vitro resistance data (GRASP), and extensive worldwide clinical experience with these agents in settings where resistance to quinolones is common. No other antimicrobials can surpass these cephalosporins in terms of the evidence base, clinical effectiveness, or favourable pharmacokinetics with respect to selection of resistance and therapeutic reserve. ${ }^{3}$ Some may question whether the adoption of cephalosporins might be a step too far when the utility of other classes of antimicrobials is not exhausted, particularly in localities where the prevalence of resistance is relatively low. The clinical care of the individual patient involves integrating the judicious use of best available evidence with clinical expertise. The revised guideline is not prescriptive but specifically acknowledges situations where use of other antibiotics may be appropriate, notably when the antimicrobial sensitivity of an isolate is known before treatment or local surveillance data suggest low prevalence of resistance to a chosen antibiotic. The oral macrolide azithromycin is specifically not recommended as a single agent treatment for gonorrhoea. Studies using a single $1 \mathrm{~g}$ dose report unpredictable treatment failures and resistance can develop during treatment. ${ }^{8}$ Unlike other antimicrobials recommended for the treatment of gonorrhoea, azithromycin MICs do not provide a reliable guide to treatment outcome and treatment failures have been reported for "susceptible" isolates. ${ }^{10}$

Other elements of the guideline were also reconsidered in the light of developing technology and changing clinical practice. The 2004 guideline includes reference to detection of $N$ gonorrhoeae by nucleic acid amplification tests (NAATS) and removal of the recommendation for a test of cure to routinely form part of post-treatment assessment. These changes may seem a significant shift from the traditional care pathway. The specialty must use its stretched resources in diagnostics, staff, and facilities to maximum effectiveness. Routine tests of cure need justification in terms of benefit to the patient or public health. Judgment on such benefits will inevitably be opinion based. Failure with recommended treatments for gonorrhoea is rare. ${ }^{11}$ There is no compelling need to prove pathogen eradication in most infections and test of cure is not recommended after treatment for genital tract chlamydial infection.

Patients with gonorrhoea deserve only to be treated according to those guidelines that reliably eradicate the infection, minimise the opportunity for the development of resistance, and meet public health needs. Any change in patient care is associated with potential benefits, risks, and costs. Realisation of the benefits of change to the guideline requires ownership and implementation by the membership of BASHH. The open debate that the revised guideline has generated has been helpful and welcomed by me and the Clinical Effectiveness Group. Guidelines are, after all, drafted from evidence filtered through opinion and clinical experience.

Sex Transm Infect 2004;80:330-331. doi: $10.1136 /$ sti.2004.012781

Correspondence to: C J Bignell, Nottingham City Hospital, Nottingham NG5 1PB, UK: cbignell@ncht.trent.nhs.uk

\section{REFERENCES}

1 Moran JS, Levine WC. Drugs of choice for the treatment of uncomplicated gonococcal infections. Clin Infect Dis 1995;20(Suppl 1):S47-65.

2 Jaffe HW, Schroeter AL, Reynolds GH, et al. Pharmacokinetic determinants in penicillin cure of gonococcal urethritis. Antimicrob Agents Chemother 1979;15:587-91.

3 Ison C, Mouton JW, Jones K, et al. Which cephalosporin for gonorrhoea? Sex Transm Infect 2004;80:386-8.

4 National Guideline on the Management of Gonorrhoea in adults. Clinical Effectiveness Group (BASHH, British Association of Sexual Health and HIV). www.bashh.org/ ceguidelines.htm.

5 GRASP Steering Group. The gonococcal resistance to antimicrobials surveillance programme (GRASP) year 2003 report. London: Health Protection Agency, 2004.

6 Fenton KA, Ison CA, Johnson AP, et al. Ciprofloxacin resistance in Neisseria 
gonorrhoeae in England and Wales in 2002. Lancet 2003;361:1867-9.

7 Ross JD, Maw R. How is gonorrhoea treated in genitourinary medicine clinics in the UK? Int J STD AIDS 2002; 13:499-500.

8 Waugh M. Open study of the safety and efficacy of a single oral dose of azithromycin for the treatment of uncomplicated gonorrhoea in men and women. J Antimicrob Chemother 1993;31(Suppl E): 193-8.

9 Young $\mathrm{H}$, Moyes A, McMillan A. Azithromycin and erythromycin resistant Neisseria gonorrhoeae following treatment with gonorrhoeae following treatment with
azithromycin. Int J STD AIDS 1997;8:299-302

10 Tapsall JW, Shultz TR, Limnios EA, et al.

Failure of azithromycin therapy in gonorrhea and discorrelation with laboratory test parameters. Sex Transm Dis 1998;25:505-8.

11 Harry TC. The management of uncomplicated adult gonococcal infection: should test of cure still be routine in patients attending genitourinary medicine clinics? Int J STD AIDS 2004; 15:453-8. issue of STI (p 342), a description of and early findings from the recently completed Chlamydia Screening Studies (ClaSS) in Bristol are presented. This study examined alternative approaches to screening using postal specimens, potential partner notification approaches, and appropriate test/specimen combinations for use in the field. Another DoH funded study examining chlamydia incidence and re-infection among screened individuals is currently under way, with results expected by end 2004. Taken in concert, these studies provide a robust evidence base to inform the initial and subsequent development of the national screening programme.

Concrete plans for a national chlamydia screening programme gained momentum with the publication of the English National Strategy for HIV and Sexual Health, ${ }^{17}$ in July 2001, which clearly outlined the government's commitment to a national roll-out of chlamydia screening. This was further strengthened by the publication of an implementation plan in June 2002, ${ }^{18}$ which confirmed the immediate implementation of screening in 10 areas in England. By September 2002, a national Chlamydia Screening Steering Group (CSSG) with multidisciplinary representation was appointed to advise the DoH on the programme's design and implementation.

The vision is to implement (by 2008) a multifaceted, evidence based, and cost effective national prevention and control programme for genital chlamydia in England, in which all sexually active adults are aware of genital chlamydia and its effects, and are able to access a range of prevention and screening services to reduce their risk of infection or onward transmission (see table 1).

The paper by LaMontagne ${ }^{2}$ describes in greater detail the objectives, phased implementation and management of the programme and results from the first year of screening. As reported in the paper in the first phase (phase 1), chlamydia screening was introduced in 10 programme areas across England, involving over 300 screening sites with a target population of over 250000 patients per annum. A further 16 areas were added in January 2004 (phase 2) leading to a total coverage of $25 \%$ of all primary care trusts (PCTs) in England 
Table 1 National Chlamydia Screening Programme, England. Screening populations and locations

\begin{tabular}{l}
\hline Opportunistic screening offered to under \\
25s attending traditional and non- \\
traditional health service settings: \\
All women and men $<25$ years old \\
attending family planning clinics, \\
contraceptive services, young people's \\
clinics, and general practices (phased \\
participation) \\
- All women $<25$ years old attending \\
antenatal, colposcopy, termination of \\
pregnancy, and community gynaecology \\
clinics \\
All women and men $<25$ years old \\
attending GUM clinics, not normally \\
receiving a sexual health screen \\
All partners of positives, regardless of \\
age \\
All women and men $<25$ years old \\
captured through non-traditional \\
screening activities - eg, university \\
medical practices, military facilities, \\
community outreach, and "pee in a pot" \\
days
\end{tabular}

(fig 1). Additional programme areas are planned for later in 2004 and successive years. The DoH pump primes the implementation of the programme to each locality providing up to $£ 150000$ for the first year, and full funding for years 2 and 3, after which all local programme costs are to be picked up by the PCT(s). The phased introduction of screening reflects DoH as well as local imperatives. One of the papers in this issue $e^{5}$ models the health care costs of this kind of screening programme and estimates the average cost (with partner management) at approximately $£ 15$ per screening offer and $£ 38$ per case identified. This model however does not address cost-effectiveness, an analysis that would require greater knowledge of outcomes.

\section{Where does this place us?}

The results of the first year of screening has clearly confirmed the feasibility of opportunistic screening approaches outside of GUM clinics and confirms the significant disease burden in this population with $10.1 \%$ positivity among women and $13.3 \%$ among men. These findings are consistent with the pilot studies $^{14} 1^{15}$ and prevalence estimates summarised in the systematic review by Adams. ${ }^{4}$ The review also provides further evidence to justify focusing chlamydia screening on people attending health care settings, rather than household screening, given the substantially lower prevalence estimates found in the latter. For example, in women under 20 years, Adams et al report overall prevalence estimates of $12.6 \%$ in antenatal clinics and $10.7 \%$ in youth

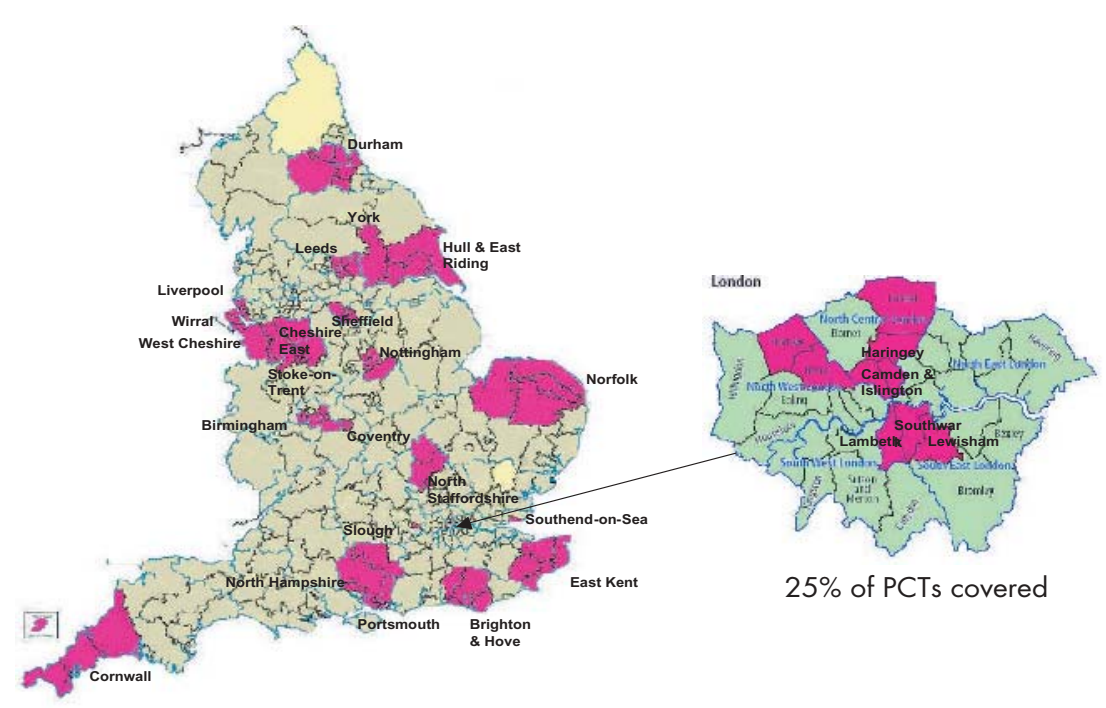

Figure 1 National Chlamydia Screening Programme, England. Phase 1 and 2 programme areas.

clinics, compared with $5.0 \%$ in population based studies. Similarly, the paper from the Class group ${ }^{3}$ describes a range of studies to inform screening methods and includes results from a pilot of active screening in which screening packs were sent to participants homes. The overall response rate of $34.3 \%$ was disappointing, with an overall prevalence of $2.8 \%$. This study highlights many of the inherent difficulties with using postal specimens, and although unsuitable as a main screening strategy, it may provide a solution for enhancing screening with particular sub-groups.

\section{CHALLENGES}

The national chlamydia screening programme is being implemented within the context of an evolving NHS characterised by devolution of decision making to the local level; heterogeneity in accountability structures and prioritisation by PCTs; and performance management by strategic health authorities. Screening in primary care will require developing a local enhanced service in GP contracts; tackling resistance to further increases in GP workload; clarification of funding for screening activity; and considerable investment in training. The results from the first year of screening nevertheless show that the implementation strategies in England can work, and a variety of non-specialist clinical settings can be enlisted to implement screening. Clearly the challenge now will be to increase coverage at all levels: nationally, within screening programme areas, and within clinical sites offering screening to their patients. Microbiology laboratories in screening areas will require additional investment and training to facilitate the use of NAATs, quality assurance and control. In areas where screening monies are being used to invest in NAATs, care is needed to avoid creating two tier services in which routine diagnostic tests are performed using lower sensitivity assays. The multisite nature of local screening activity, with some sites still not computerised, requires overcoming dataflow logistics and centralising data management. Care will be required not to overload GUM clinics with those screened positive for genital chlamydial infection, and new models of care for managing. These patients and their contacts in the community (either at a local chlamydia office or at screening sites) will need to be evaluated. Finally, as and when local programmes become established, performance management and quality assurance arrangements will be required to ensure that chlamydia screening activity is maintained over time.

Finally, these and other studies confirm the high prevalence of asymptomatic genital chlamydial infection in men and the difficulties opportunistic methods have in identifying and testing them. Although the national chlamydia screening programme actively includes men and women in all screening activities, effective strategies need to be developed, evaluated and good practice shared. Greater effort will be needed to ensure that men themselves are aware of chlamydia, its effects and strategies for its prevention and control. In this regard, the programme has the potential to make a substantial contribution to improving men's sexual health in the coming years.

These factors aside, the chlamydia screening programme as envisioned in England represents a bold and considered move towards controlling this 
devastating sexually transmitted infection. The phased implementation will allow further refinement of the structure and process of the screening programme over the coming months; however, successful implementation will only be achieved with a sustained commitment to joint working among stakeholders at local and national levels.

\section{ACKNOWLEDGEMENTS}

All members of the Chlamydia Advisory Group contributed to the preparation of this editorial.

Sex Transm Infect 2004;80:331-333.

doi: $10.1136 /$ sti.2004.009787

\section{Authors' affiliations}

K A Fenton, HIV and Sexually Transmitted Infections Department, Health Protection Agency, London, UK

H Ward, Department of Infectious Disease Epidemiology, Imperial College London

Correspondence to: Dr Kevin A Fenton, HIV and Sexually Transmitted Infections Department, Health Protection Agency, Communicable Disease Surveillance Centre, 61 Colindale Avenue, London NW9 5EQ, UK; Kevin.fenton@hpa.org.uk

\section{REFERENCES}

1 House of Commons. Select Committee on Health. Third report on sexual health. Available at www.parliament.the-stationery-office.co.uk/pa/ cm200203/cmselect/cmhealth/69/6902.htm Last accessed on 1 March 2004.

2 LaMontagne DS, Fenton KA, Randall S, et al. Establising the National Chlamydia Screening Programme in England: results from the first full year of screening. Sex Transm Infect 2004;80:335-41.

3 Low N, McCarthy A, Macleod J, et al. The chlamydia screening studies: rationale and design. Sex Transm Infect 2004;80:342-8.

4 Adams EJ, Charlett A, Edmunds WJ, et al. Chlamydia trachomatis in the United Kingdom: a systematic review and analysis of prevalence studies. Sex Transm Infect 2004:80:354-62.

5 Adams EJ, LaMontagne DS, Johnston AR, et al. Modelling the heathcare costs of an opportunistic Chlamydia screening programme. Sex Transm Infect 2004;80:362-70.

6 Department of Health. The national chlamydia screening programme in England, Programme overview, core requirements and data collection. London: DoH, April 2004;(2)

7 Health Protection Agency, SCIEH, ISD, National Public Health Service for Wales, CDSC Northern Ireland, the UASSG. Renewing the focus. HIV and other sexually transmitted infections in the United Kingdom in 2002. London: Health Protection Agency, November 2003.

8 Additional information on the epidemiology of genital chlamydia infections diagnosed in GUM clinics in the United Kingdom is available at www. hpa.org.uk/infections/topics_az/hiv_and_sti/ sti-chlamydia/epidemiology/epidemiology.htm.

9 Morre SA, van den Brule AJC, Rozendaal L, et al. The natural course of asymptomatic Chlamydia trachomatis infections: $45 \%$ clearances and no development of clinical PID after one-year follow-up. Int J STD AIDS 2002;

13(Suppl 2):12-18.

10 Cates W, Wasserheit JN. Genital chlamydial infections: epidemiology and reproductive sequelae. Am J Obstet Gynecol 1991; 164(6 P+ 2):1771-81

11 Honey E, Augood C, Templeton A, et al. Cost effectiveness of screening for Chlamydia trachomatis: a review of published studies. Sex Transm Infect 2002;78:406-12.

12 Chief Medical Officer's Expert Advisory Group. Main report of the CMO's expert advisory group on Chlamydia trachomatis. London: Department of Health, 1998.

13 Department of Health. Chlamydia Screening Pilot: Report of 1999-2000 study. London: DoH, 2002.

14 Pimenta JM, Catchpole M, Rogers PA, et al. Opportunistic screening for genital chlamydial infection I: Acceptability of urine testing in primary and secondary healthcare settings. Sex Transm Infect 2003:79:16-21.

15 Pimenta JM, Catchpole M, Rogers PA, et al. Opportunistic screening for genital chlamydial infection II: Prevalence among health care attenders, outcome and evaluation of positive cases. Sex Transm Infect 2003;79:22-7.

16 Fenton K, Korovessis C, Johnson AM, et al. Sexual behaviour in Britain: reported sexually transmitted infections and prevalence genital Chlamydia trachomatis infection. Lancet 2001;358:1851-4

17 Department of Health. The national strategy for sexual health and HIV. London: DoH, July 2001.

18 Department of Health. The national strategy for sexual health and HIV-implementation action plan. London: DoH, June 2002.

\section{Current status of syndromic management of sexually transmitted infections in developing countries}

\section{B Vuylsteke}

The syndromic approach has been a major step forward in rationalising and improving management of STI

D lagnosis of a presumed sexually transmitted infection (STI) has traditionally been based on either clinical diagnosis, which is often inaccurate and incomplete, or laboratory diagnosis, which is complex, very expensive, and may delay treatment. As early as the 1970s, public health physicians, particularly those working in Africa, became interested in testing simple clinical tools for controlling and treating STIs. ${ }^{1}$ This resulted in the design and promotion of "syndromic management" guidelines for STIs by the World Health Organization in 1991. ${ }^{2}$ The syndromic approach does not require identification of the underlying aetiology. Instead, it is based on the identification of a syndrome-that is, a group of symptoms and easily recognised signs associated with a number of well defined aetiologies. Treatment is provided for the majority of the organisms locally responsible for the syndrome.

It rapidly became clear that the syndromic approach offered enormous advantages compared to the traditional approach, although more evidence was needed to rationalise and convince policy makers. ${ }^{3}$ Algorithms based on a syndromic approach were evaluated in many different settings, results of which were reported in the late 1990s-for example in a supplement of STI. ${ }^{4}$ In a study in South Africa, for instance, the syndromic management protocols provided adequate treatment for more than $90 \%$ of patients with genital ulcer syndrome (GUS). ${ }^{5}$ In another study in Indonesia, the positive predictive value (PPV) of a syndromic approach for gonococcal and/or chlamydial urethritis was between $75 \%$ and $97 \%$, resulting in a low cost per real case treated. ${ }^{6}$ In addition, the cure rate for urethral discharge with the syndromic approach was 99\%. ${ }^{6}$ In order to decrease the number of women who would be treated unnecessarily for cervical infections, a risk assessment was incorporated into the syndromic approach to vaginal discharge. As a result, a woman with a complaint of vaginal discharge is treated systematically for vaginal infections, but only if her risk assessment is positive will she receive treatment for gonococcal and chlamydial infection as well. Using a risk score assessment in Tanzania, the overtreatment rate for cervical infections decreased from 92\% to $17 \%$ in pregnant women and from $89 \%$ to $36 \%$ in non-pregnant women with vaginal discharge. ${ }^{7}$ By the late 1990s, the syndromic approach was largely promoted and used worldwide, and not only in developing countries.

There is enough evidence now that the syndromic approach is effective and has had an impact on the STI epidemic. Dramatic declines in STI rates have been observed following control strategies based on the syndromic approach, such as in sex workers in Côte d'Ivoire, Senegal and South Africa, and in STI 


\section{Key messages}

- The syndromic approach has been a major step forward in rationalising and improving management of STI

- The performance of genital ulcer syndrome (GUS) treatment flow charts depends on the aetiological patterns of GUS in different settings

- The risk score approach should not be used as an STI screening tool or diagnostic test in asymptomatic or poorly symptomatic women

- Simple and rapid point of care tests may contribute to improve STI care for women in the near future

clinics in Kenya and in Burkina Faso. ${ }^{8-10}$ The studies in Mwanza (Tanzania) and Masaka (Uganda) demonstrated the impact of syndromic management beyond the STI clinic attendees they targeted by decreasing STI prevalences in the general population: serological syphilis by $20 \%$ and male urethritis by $50 \%$ in Mwanza, and gonorrhoea by $70 \%$ in Masaka. ${ }^{12}$ The declining prevalence of bacterial infections in some of the key syndromes in parts of Africa is a testimony to the success of widespread syndromic management use. ${ }^{9}$

In this issue of STI ( $\mathrm{p}$ 392, Wolday et al describe the results of a study on risk factors associated with the failure of syndromic management of STIs among women seeking treatment in a primary healthcare centre in Addis Ababa. Syndromic treatment did not result in clinical improvement in $30 \%$ of the women, and the GUS was significantly associated with treatment failure. The authors argue that the treatment failure is probably a result of the high proportion of ulcers caused by herpes simplex type 2 virus (HSV-2) in this high HIV prevalence setting. The performance of syndromic treatment flow charts depends on the aetiological patterns of the syndrome, and herpes is not addressed by the former WHO algorithms. ${ }^{13}$ The syndromic approach became victim of its own success; because of the improved control of chancroid and syphilis in some regions it has become apparent that the GUS, particularly in the sub-Saharan countries, is more frequently caused by HSV2 infections. The WHO is currently recommending including the treatment for HSV-2 in the management of genital ulcers, especially in settings where HSV2 prevalence is $30 \%$ or higher. ${ }^{13}$ Adding aciclovir to the syndromic treatment of ulcers, however, will not necessarily lead to higher cure rates.

Another area of concern is the use of the syndromic management in low STI prevalence settings, especially when the approach is used as a screening tool. ${ }^{14} 15$ It should be stressed that the syndromic approach was developed as a diagnostic tool in symptomatic patients, it was never meant to be a screening tool. Traditionally, screening tools are used to minimise the number of (more expensive) standard diagnostic tests by identifying a group of people with a higher than average prevalence of infection. In the absence of such a test, the risk score approach should not be used as a substitute for standard diagnosis because of its poor discriminative ability. The current picture may change, however, when simple, cheap, and rapid diagnostic tests for Neisseria gonorrhoeae and Chlamydia trachomatis are available in developing countries. The development of such tests is considered by STI control programme managers and STI specialists to be an absolute priority in STI research. Major progress has recently been made in this field. A rapid (25 minute), cheap (\$US 0.85) dipstick for chlamydial infection "Firstburst" has been developed recently and is awaiting FDA approval. Another duplex ( $N$ gonorrhoeae and $C$ trachomatis) test is undergoing evaluation. ${ }^{16}$ These tests may represent an important breakthrough for STI control in symptomatic and asymptomatic women in developing countries.

In conclusion, the syndromic approach has been a major step forward in rationalising and improving management of STI, and its impact on the STI epidemic has been observed in various settings. However, syndromic algorithms have some shortcomings, and they should be periodically revised and adapted to the epidemiological patterns of STI in a given setting. Simple and rapid point of care tests might help the screening of asymptomatic and low symptomatic women and the diagnosis of STI in symptomatic women. Finally, we should not forget that many other factors play a part in the successful control of STIs, including availability of effective and affordable drugs, accessible and acceptable health services, training and supervision of healthcare workers, and behavioural interventions to prevent new infections by promoting safer sex. ${ }^{17} 18$
Sex Transm Infect 2004;80:333-334. doi: $10.1136 /$ sti.2004.009407

Correspondence to: Dr Bea Vuylsteke, Institute of Tropical Medicine, Antwerp, Belgium; bvuylsteke@itg.be

\section{REFERENCES}

1 Meheus AZ. Practical approaches in developing nations. In: Holmes KK, Mardh P-A, Sparling PF, et al. Sexually transmitted diseases. 1 st ed. New York: McGraw-Hill, 1984:998-1008.

2 World Health Organization. Guidelines for the management of sexually transmitted infections. Geneva: WHO, WHO/HIV_AIDS/2001, 01.

3 Lush L, Walt G, Ogden J. Transferring policies for treating sexually transmitted infections: what's wrong with global guidelines? Health Policy and Planning 2003; 18:18-30.

4 STI. Syndromic approach to STD management. Sex Transm Infect 1998;74(Suppl 1).

5 Hiun Y, Morse SA, Dangor Y, et al. Comparison of clinically directed, disease specific, and syndromic protocols for the management of genital ulcer disease in Lesotho. Sex Transm Infect 1998;74(Suppl 1):S23-8.

6 Diajakusumah T, Sudigdoadi S, Keersmaekers K, et al. Evalution of syndromic patient management algorithms for urethral discharge. Sex Transm Infect 1998;74(Suppl 1):S29-33.

7 Mayaud P, ka-Gina G, Cornelissen J, et al. Validation of a WHO algorithm with risk assessment for the clinical management of vaginal discharge in Mwanza, Tanzania. Sex Transm Infect 1998;74(Suppl 1):S77-84.

8 Ghys PD, Diallo MO, Ettiegne-Traore V, et al. Increase in condom use and decline in HIV and sexually transmitted diseases among female sex workers in Abidjan, Cote d'Ivoire, 1991-1998. AIDS 2002;16:251-8.

9 Steen R. Eradicating chancroid. Bull World Health Organ 2001;79:818-26.

10 Nagot N, Meda N, Ouangre A, et al. Review of STI and HIV epidemiological data from 1990 to 2001 in urban Burkina Faso: implications for STI and HIV control. Sex Transm Infect 2004;80: 124-9.

11 Mayaud P, Mosha F, Todd J, et al. Improved treatment services significantly reduce the prevalence of sexually transmitted diseases in rural Tanzania: results of a randomized controlled trial. AIDS 1997;11:1873-80.

12 Kamali A, Quigley M, Kakiyingi J, et al. Syndromic management of sexually-transmitted infections and behaviour change interventions on transmission of HIV-1 in rural Uganda: a community randomised trial. Lancet 2003;361:645-52.

13 World Health Organization. Report of the consultation meeting on improving management of sexually transmitted diseases. Geneva: WHO, 28-30 Nov, 2001.

14 Hawkes SJ, Morison L, Foster S, et al. Reproductive tract infections in women in lowincome, low-prevalence situations: assessment of syndromic management in Matlab, Bangladesh. Lancet 1999;354:1776-81.

15 Kapiga SH, Vuylsteke B, Lyamya EF, et al. Evaluation of sexually transmitted diseases diagnostic algorithms among family planning clients in Dar es Salaam, Tanzania. Sex Transm Infect 1998;74(Suppl 1):S132-8.

16 Peeling R. Sexually transmitted diseases diagnostics initiative (SDI). Presented at the consultation meeting on improving management of sexually transmitted diseases. Geneva: WHO, 28-30 Nov, 2001.

17 Buvé A, Changalucha J, Mayaud P, et al. How many patients with a sexually transmitted infection are cured by health services? A study from Mwanza region; Tanzania. Trop Med Int Health 2001;6:971-9.

18 UNAIDS/WHO. Sexually transmitted diseases: policies and principles for prevention and care. Geneva: UNAIDS, 1999, UNAIDS/ $01.11 \mathrm{E}$. 